\title{
Identification of differentially expressed proteins in the gastric mucosal atypical hyperplasia tissue microenvironment
}

\author{
HE-LIANG ZHANG ${ }^{1,2}$, CHONG-YUAN LIU ${ }^{1}$, WEI MA ${ }^{1}$, LIN HUANG $^{3}$, \\ CHANG-JIAN LI ${ }^{4}$, CHENG-SONG LI ${ }^{4}$ and ZHI-WEI ZHANG ${ }^{1}$ \\ ${ }^{1}$ Key Laboratory of Cancer Cellular and Molecular Pathology, Cancer Research Institute of Medical College, \\ University of South China, Hengyang, Hunan 421001; ${ }^{2}$ Medical Company, Troops 66028 of People's Liberation Army, \\ Chengde, Hebei 067000; ${ }^{3}$ Department of Pediatrics, Shaoyang Medical School, Shaoyang, Hunan 422000; \\ ${ }^{4}$ Clinical Medicine Undergraduate Program, Medical College, University of South China, \\ Hengyang, Hunan 421001, P.R. China
}

Received October 15, 2017; Accepted April 5, 2018

DOI: $10.3892 / \mathrm{ol} .2018 .8941$

\begin{abstract}
In the present study, the interaction of proteins in the microenvironment of gastric mucosal atypical hyperplasia was analyzed. The stromata of normal gastric mucosa (NGM) and gastric mucosal atypical hyperplasia (GMAH) tissues were purified with laser capture microdissection (LCM). The differentially expressed GMAH proteins of the NGM and GMAH tissues were identified by quantitative proteomic techniques with isotope labeling. The cross-talk between differentially expressed proteins in NGM and GMAH tissues was then analyzed by bioinformatics. There were 165 differentially expressed proteins identified from the stromata of NGM and GMAH tissues. Among them, 99 proteins were upregulated and 66 were downregulated in GMAH tissue. The present study demonstrated that these proteins in gastric mucosal atypical hyperplasia were involved in cancer-associated signaling pathways, including the p53, mitogen-activated protein kinase (MAPK), cell cycle and apoptosis signaling pathways, and were involved in cellular growth, cellular proliferation, apoptosis and the humoral immune response. The results of the present study suggest that the 165 differentially expressed proteins, including S100 calcium-binding protein A6 (S100A6) and superoxide dismutase 3 (SOD3) in the microenvironment of gastric mucosal atypical hyperplasia, are involved in the p53, MAPK, cell cycle and apoptosis signaling pathways, and serve a function in the pathogenesis of gastric cancer.
\end{abstract}

Correspondence to: Dr Zhi-Wei Zhang, Key Laboratory of Cancer Cellular and Molecular Pathology, Cancer Research Institute of Medical College, University of South China, 28 Changsheng Road, Hengyang, Hunan 421001, P.R. China

E-mail: nhdxzzw@qq.com

Key words: gastric mucosal atypical hyperplasia, microenvironment, protein expression

\section{Introduction}

Gastric carcinoma (GC), a serious threat to human health, is one of the most common malignancies in China, and its incidence and deaths rank first in the digestive system in 2015 (1). The occurrence of GC involves a complex pathological process associated with polygenic interactions and multi-phase evolution (2). The majority of patients experience the typical stages of normal gastric mucosa, chronic atrophic gastritis, precancerous lesions (atypical hyperplasia of gastric mucosa and intestinal metaplasia), early stages of gastric cancer, and advanced stage of disease (3). However, at present, the molecular mechanisms underlying the occurrence of $\mathrm{GC}$ remain unclear.

The cross-talk that exists between tumor cells and the microenvironment serves an important function in the occurrence and development of tumors (4). Tumor cells adapt to their microenvironment and exhibit corresponding biological characteristics. The tumor microenvironment refers to the internal environment in which the tumor grows, which is primarily composed of various interstitial cells, blood vessels, nerves, interstitial fluid and a small number of leucocytes (5). Tumor cells are able to induce mesenchymal cells to produce a variety of cytokines and growth factors that promote tumorigenesis and development (6). According to previous studies (7), it is possible to target the formation mechanism of the tumor microenvironment in order to prevent the proliferation and metastasis of tumor cells. Knowledge of the interaction between the microenvironment and tumor cells is expected to provide a rich theoretical basis for the treatment of tumors. The aim of the present study was to elucidate the molecular mechanisms underlying the occurrence of GC by analyzing the protein interactions in gastric mucosal atypical hyperplasia.

\section{Materials and methods}

Tissue samples. Matching specimens, including 20 cases of normal gastric mucosa (NGM) tissue and gastric mucosa atypical hyperplasia (GMAH) tissue, were collected from 
The First Affiliated Hospital of University of South China between September 2016 and June 2017. The Cancer Research Institute of University of South China and The First Affiliated Hospital of University of South China are cooperative relations. Researchers from Cancer Research Institute are permitted to travel to the hospital and collect specimens with the permission of the medical ethics committee of University of South China. Specimens were collected from the stomach within 5 min of resection, and the gastric mucosal surface was washed with physiological saline prior to and following the incision. The samples were immediately frozen in liquid nitrogen and stored at $-80^{\circ} \mathrm{C}$. Table I presented the clinical data including tumor stage determined by the eighth edition AJCC cancer staging manual (8) of 20 patients with GC. Two senior professional pathologists from Cancer Research Institute of University of South China were asked to independently diagnose the collected tissue samples without knowing any clinical or pathological data.

Ethics statement. The human GC tissue samples were collected from The First Affiliated Hospital of University of South China according to the institutional and governmental guidelines. All patients involved in the present study provided written informed consent, and the present study was approved by the medical ethics committee of University of South China (Hengyang, China).

Preparation and staining of frozen sections. The tissue samples were removed from liquid nitrogen and placed on a cryostat device carrier (Leica Biosystems GmbH, Wetzlar, Germany). Following the addition of optimal cutting temperature compound (OCT) embedding agent (Leica Microsystems $\mathrm{GmbH}$ ), the samples were frozen at $-25^{\circ} \mathrm{C}$ for $20 \mathrm{~min}$. Next, the samples were immobilized to the platform of the cryostat device, and frozen sections were made at a thickness of $8 \mu \mathrm{m}$. The frozen sections were affixed to film slides (Leica Microsystems $\mathrm{GmbH}$ ) pretreated with ultraviolet (UV) light. Finally, the slides were fixed with $75 \%$ ethanol at $4^{\circ} \mathrm{C}$ for $60 \mathrm{sec}$, stained with $0.5 \%$ methyl green (Sigma-Aldrich; Merck $\mathrm{KGaA}$, Darmstadt, Germany) at $4^{\circ} \mathrm{C}$ for $30 \mathrm{sec}$ and discolored with $95 \%$ ethanol at $4^{\circ} \mathrm{C}$ for $5 \mathrm{sec}$.

Laser capture microdissection (LCM). The frozen tissue sections stained with methyl green were placed on an LCM apparatus (Leica LMD6, Leica Microsystems $\mathrm{GmbH}$ ) platform. The target tissue was outlined on the display, and the laser automatically cut the target tissue in the slice. Dissolved one tablet of protease inhibitor cocktail tablets (Roche Diagnostics, Basel, Switzerland) in $50 \mathrm{ml}$ ultrapure water to prepare 5\% working solutions. The tissues were collected in a tube containing 2-3 $\mu \mathrm{l}$ protease inhibitor working solutions and were frozen at $-80^{\circ} \mathrm{C}$ for later use.

Protein extraction and isobaric tags for relative and absolute quantitation (iTRAQ) isotope labeling. The mesenchyma of the NGM and GMAH tissues were extracted using a lysis buffer (10 mM PMSF, $65 \mathrm{mM}$ dithiothreitol, $7 \mathrm{M}$ urea and $2 \mathrm{M}$ thiourea) (GE Healthcare Life Sciences, Little Chalfont, UK) and centrifuged at $4^{\circ} \mathrm{C}, 12,000 \mathrm{x}$ for $30 \mathrm{~min}$. The supernatant included the total proteins of the NGM and GMAH mesenchyma. The total proteins were extracted and quantified using a bicinchonic acid protein assay kit (Beyotime Institute of Biotechnology, Shanghai, China), according to the manufacturer's protocol. The total proteins of the NGM mesenchyma were labeled with iTRAQ reagent 114; total proteins of the GMAH mesenchyma were labeled with iTRAQ reagent 118 (both Applied Biosystems; Thermo Fisher Scientific, Inc., Waltham, MA, USA) according to the manufacturer's protocol. A total of $100 \mu 1$ ultrapure water was used to end the reaction. All protein samples were homogenized and lyophilized, and then the samples were dissolved in deionized water containing $0.1 \%$ formic acid (FA; Tedia Company, Fairfield, OH, USA). The marked samples were eluted twice with Sep-Pak $\mathrm{C}_{18} 1 \mathrm{cc}$ Vac cartridges (Waters Corporation, Milford, MA, USA) with deionized water containing $0.1 \%$ FA and then once with 50\% acetonitrile (ACN) (Thermo Fisher Scientific, Inc.) containing 0.1\% FA. The cleaning solution was collected and lyophilized.

Identification of differentially expressed proteins. The samples marked with iTRAQ were dissolved in $1 \mathrm{ml}$ strong cation-exchange (SCX) buffer [25\% (v/v) ACN and $10 \mathrm{mM}$ $\mathrm{KH}_{2} \mathrm{PO}_{4}, \mathrm{pH}$ 2.6] for SCX separation. The two samples containing mesenchymal proteins of NGM and GMAH were mixed and loaded into a polysulfoethyl column and segregated using a 20AD high performance liquid chromatography (HPLC) system (Shimadzu Corporation, Kyoto, Japan) with the following conditions: i) $10 \mathrm{mM} \mathrm{K \textrm {K } _ { 2 }} \mathrm{PO}_{4}$ and $25 \% \mathrm{ACN}$, pH 2.6; ii) $10 \mathrm{mM} \mathrm{KH} \mathrm{PO}_{4}, 350 \mathrm{mM} \mathrm{KCl}$ and $25 \% \mathrm{ACN}$, $\mathrm{pH}$ 2.6. The following settings were used: UV detection wavelength: $214 / 280 \mathrm{~nm}$; flow rate: $200 \mu \mathrm{l} / \mathrm{min}$ for $60 \mathrm{~min}$; salt gradient: from $5 \%$ i) at $5 \mathrm{~min}$ to $25 \%$ ii) at $40 \mathrm{~min}$. Next, the products were concentrated by vacuum centrifugation for reverse-phase HPLC-mass spectrometry (MS) analysis. The samples were dissolved in $50 \mu 15 \% \mathrm{ACN}$ containing $0.1 \% \mathrm{FA}$ and were loaded into a Zorbax 300SB- $\mathrm{C}_{18}$ column (Agilent Technologies, Inc., Santa Clara, CA, USA). The conditions were as follows: i) $5 \% \mathrm{ACN}, 0.1 \% \mathrm{FA}$; ii) $95 \% \mathrm{ACN}, 0.1 \% \mathrm{FA}$. Flow rate: $300 \mathrm{nl} / \mathrm{min}$ for $90 \mathrm{~min}$. Salt gradient: from $5 \%$ i) at $5 \mathrm{~min}$ to $35 \%$ ii) at $70 \mathrm{~min}$. The data were analyzed using QSTAR-XL (Applied Biosystems; Thermo Fisher Scientific, Inc.) and tandem MS (MS/MS). Finally, the IPI human database (version 3.45; URL: http://www.ebi.ac.uk/IPI) was searched for protein information, and the confidence level was set to be $>95 \%$, and the ion peak areas of $\mathrm{m} / \mathrm{z} 114$ and 118 were integrated to perform relative quantitative analysis of proteins.

Western blot analysis. The total NGM and GMAH mesenchymal proteins were mixed with $5 \mathrm{X}$ loading buffer (Beyotime Institute of Biotechnology) and boiled for $5 \mathrm{~min}$. The proteins had been quantified using a bicinchonic acid protein assay kit (Beyotime Institute of Biotechnology). Next, the samples were separated using $10 \%$ gradient SDS-PAGE gels at $30 \mu \mathrm{g}$ per lane and transferred onto PVDF membranes (Merck KGaA). The membranes were blotted with 5\% fat-free milk suspended in TBST at room temperature for $1 \mathrm{~h}$, incubated at $4^{\circ} \mathrm{C}$ overnight with S100 calcium-binding protein A6 (S100A6) antibody (1:1,000) (sc-53950; Santa Cruz Biotechnology, Inc., Dallas, TX, USA) and superoxide dismutase 3 (SOD3) antibody $(1: 1,000)$ (sc-58427; Santa Cruz Biotechnology, Inc.), washed 
Table I. Clinicopathological features of patients with gastric cancer.

\begin{tabular}{|c|c|c|c|c|c|}
\hline No. & Sex & Age, years & Differentiation & Tumor stage (8) & Date of collection \\
\hline 1 & Male & 49 & Moderate & II & September 2016 \\
\hline 2 & Female & 64 & Poor & IV & September 2016 \\
\hline 3 & Female & 69 & Poor & IV & September 2016 \\
\hline 4 & Male & 62 & Poor & II & October 2016 \\
\hline 5 & Male & 44 & Moderate & II & October 2016 \\
\hline 6 & Male & 60 & Poor & II & November 2016 \\
\hline 7 & Male & 53 & Poor & II & November 2016 \\
\hline 8 & Female & 40 & Poor & III & November 2016 \\
\hline 9 & Male & 67 & Poor & II & December 2016 \\
\hline 10 & Female & 81 & Poor & II & December 2016 \\
\hline 11 & Female & 47 & High & II & January 2017 \\
\hline 12 & Male & 63 & Poor & II & February 2017 \\
\hline 13 & Male & 52 & Poor & IV & March 2017 \\
\hline 14 & Male & 46 & Moderate & II & March 2017 \\
\hline 15 & Male & 51 & Poor & II & March 2017 \\
\hline 16 & Male & 60 & Moderate & II & March 2017 \\
\hline 17 & Female & 66 & Poor & III & April 2017 \\
\hline 18 & Male & 67 & Poor & II & April 2017 \\
\hline 19 & Female & 68 & Poor & II & May 2017 \\
\hline 20 & Female & 62 & Poor & III & June 2017 \\
\hline
\end{tabular}

and then incubated with goat anti-mouse IgG-HRP $(1: 2,000)$ (sc-2005; Santa Cruz Biotechnology, Inc.) at room temperature for $2 \mathrm{~h}$. Detection of immunoreactivity was achieved using enhanced chemiluminescence (GE Healthcare Life Sciences).

Immunohistochemistry. The present study used S-P immunohistochemical staining kits (MXB Company, Fujian Province, China; URL: http://www.maxim.com.cn/). The NGM and GMAH tissues were fixed with $10 \%$ formalin and embedded in paraffin. The expression of S100A6 and SOD3 proteins were detected according to the manufacturer's protocol. Briefly, 4- $\mu$ m-thick sections were prepared and mounted on poly-L-lysine-coated glass slides, air-dried, deparaffinized with xylene and rehydrated in a descending ethanol series. Following microwave treatment for $20 \mathrm{~min}$, endogenous peroxidase activity was suppressed using $0.3 \%$ hydrogen peroxide. The sections were treated with $5 \%$ normal goat serum (SL038) (Solarbio Life Sciences, Tongzhou Dist. Beijing, China) at room temperature for $15 \mathrm{~min}$ to block non-specific binding. The sections were incubated with anti-S100A6 (1:100) or anti-SOD3 (1:100) antibody overnight at $4^{\circ} \mathrm{C}$, and then incubated with goat anti-mouse IgG-FITC (1:200) (sc-2010; Santa Cruz Biotechnology, Inc.) at room temperature for $60 \mathrm{~min}$ followed by horseradish peroxidase-labeled streptavidin for $5 \mathrm{~min}$ at room temperature. The sections were counterstained with $0.1 \%$ hematoxylin at room temperature for $30 \mathrm{sec}$. The tissue staining was observed under a light microscope at a magnification of $x 40$. The final immunoreactive score was based on protein staining intensity and the percentage of positive cells. Staining intensity was defined as 1 (negative), 2 (yellow) and 3 (brown). The percentage of positive cells was defined as $1(<10 \%$ positive cells), 2 (11-50\% positive cells) and 3 ( $>50 \%$ positive cells). The final immunoreactive score was calculated as: Staining intensity $x$ percentage of positive cells. The classification of the final score was defined as - (score 1), + (score 2-4) and $+++($ score $>4)$.

Protein signaling pathways and interaction analysis. Visant software (version 3.91; URL: http://visant.bu.edu) was used to analyze the interactions between proteins. Additionally, the network of direct interactions between proteins was analyzed. The Clue Gene Ontology (GO), Kyoto Encyclopedia of Genes and Genomes (KEGG) analysis of protein signaling pathways was performed using Cytoscape software (version 2.8.2; URL: http://www.cytoscape.org). GO_BP, GO_CC and GO_MF analyses were executed with David Functional Annotation (URL: http://david.abcc.ncifcrf.gov).

Statistical analysis. The data are reported as the mean \pm standard deviation. Statistical analysis was performed using SPSS statistical package (version 18.0; SPSS, Inc., Chicago, IL, USA) as follows: Comparison between individual subgroups was performed using the Mann-Whitney U test, and correlation analysis between groups was performed using Spearman's rank correlation test. $\mathrm{P}<0.05$ was considered to indicate a statistically significant difference.

\section{Results}

Purified mesenchyma of NGM and GMAH tissues. The NGM and GMAH tissues were obtained from fresh specimens of GC following surgical resection, and all tissues were confirmed by pathology. The mesenchyma of NGM and GMAH tissues were 

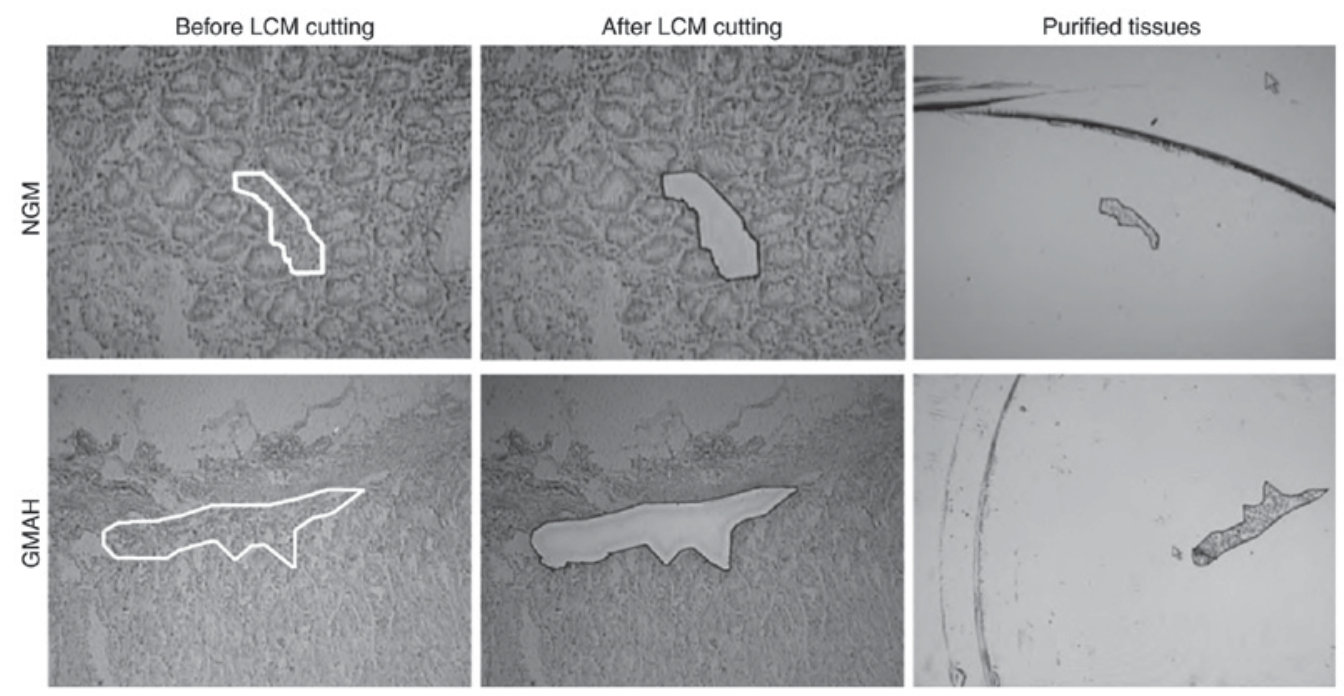

Figure 1. Purification of mesenchyma of NGM and GMAH tissues by LCM. The purified mesenchyma of NGM and GMAH tissues prior to and following LCM are indicated in the area surrounded by the closed line at a magnification of x10. NGM, normal gastric mucosa; GMAH, gastric mucosal atypical hyperplasia; LCM, laser-capture microdissection.

purified by LCM (Fig. 1). The purity of objective groups was $>95 \%$.

Identification of differentially expressed proteins. The NGM and GMAH mesenchyma proteins were divided into solutions and marked using different isotopic iTRAQ. Next, the NGM and GMAH proteins were separated using a 20AD HPLC system and identified using QSTAR-XL MS/MS. A total of 165 differentially expressed proteins between the NGM and GMAH mesenchyma were identified (Table II). The G/N value (NGM/GMAH tissue) was determined as the mean protein expression level. In total, 99 proteins $(\mathrm{G} / \mathrm{N}>1.5)$ were identified to be upregulated and 66 proteins $(\mathrm{G} / \mathrm{N}<0.667)$ were identified to be downregulated in the GMAH mesenchyma. The expression levels of the S100A6 and SOD3 proteins were different in the mesenchyma of the NGM and GMAH tissues, and were associated with tumorigenesis in previous studies (9,10). Fig. 2 presents the MS results and the quantification of the S100A6 (Fig. 2A) and SOD3 proteins (Fig. 2B).

S100A6 is upregulated and SOD3 is downregulated in GMAH mesenchymal tissue. The 20 samples of NGM and GMAH tissues were collected and purified. Next, the tissues were sectioned. The expression levels of the S100A6 and SOD3 proteins in NGM and GMAH mesenchyma were detected using western blotting and immunohistochemistry. The result of western blotting indicated that the S100A6 protein was upregulated, but that the SOD3 protein was significantly downregulated in the GMAH mesenchyma when compared with the NGM tissue ( $\mathrm{P}<0.01$; Fig. 3A). Immunohistochemistry analysis demonstrated that S100A6 and SOD3 proteins were expressed in the mesenchyma of NGM and GMAH tissues; however, the staining intensity and expression levels of the S100A6 protein in the GMAH tissue were increased compared with those in the NGM tissue. The expression of the SOD3 protein was the opposite (Fig. 3B and C). Therefore, the S100A6 and SOD3 expression levels were significantly different between the NGM and GMAH tissues $(\mathrm{P}<0.05$; Table III). These results were consistent with the results of quantitative proteomics in the present study (Table II).

Interaction of differentially expressed proteins and relevant signaling pathways analysis. The interaction between 165 differentially expressed proteins in GMAH were analyzed using Visant software. It was identified that 140 proteins acted as network nodes and interacted with each other. The results of KEGG signal pathway analysis demonstrated that the 165 proteins were involved in a number of tumor signaling pathways, including the $\mathrm{p} 53$, mitogen-activated protein kinase (MAPK), cell cycle, and apoptosis signaling pathways (Fig. 4). Next, the biological functions of the 165 proteins were analyzed with the David tool, which indicated that the proteins were involved in cell growth, proliferation, apoptosis and the humoral immune response (results not shown).

\section{Discussion}

The microenvironment is composed of stromal cells, immune cells and cytokines, and the tumor microenvironment has been proven to determine the biological behavior of tumor cells $(11,12)$. It is hypothesized that the interactions of protease, cytokines and receptors in the tumor microenvironment affect the osmotic pressure and metabolism of the tumor, which may result in immune escape and neoplasia $(13,14)$. It is important to monitor cell behavior and prevent cancer by understanding changes in the microenvironment, which serve important functions in tumor occurrence and development (4). In the present study, 165 proteins that were differentially expressed between the NGM and GMAH tissue microenvironments were screened. These proteins were demonstrated to be involved in signaling pathways associated with cancer, including the MAPK, VEGF and p53 signaling pathways, suggesting that these proteins may regulate cell growth, proliferation, apoptosis and the humoral immune response. However, the interaction network should be further characterized in follow-up studies. In the present study, the 
Table II. Differentially expressed proteins between the NGM and GMAH mesenchyma.

\begin{tabular}{|c|c|c|c|}
\hline No. & Accession no. & Protein name & GMAH vs. NGM \\
\hline 1 & IPI00872780.1 & ANXA4, annexin A4 & $\uparrow 1.5704$ \\
\hline 2 & IPI00027230.3 & HSP90B1, endoplasmin precursor & $\uparrow 1.5704$ \\
\hline 3 & IPI00024920.1 & ATP5D, ATP synthase subunit $\delta$ & $\uparrow 1.5704$ \\
\hline 4 & IPI00013508.5 & ACTN1, $\alpha$-actinin-1 & $\uparrow 1.5848$ \\
\hline 5 & IPI00216135.1 & TPM1, isoform 3 of tropomyosin $\alpha-1$ chain & $\uparrow 1.5995$ \\
\hline 6 & IPI00788802.1 & TKT, transketolase variant & $\uparrow 1.6292$ \\
\hline 7 & IPI00647915.1 & TAGLN2, $24 \mathrm{kDa}$ protein & $\uparrow 1.6292$ \\
\hline 8 & IPI00025874.2 & RPN1 & $\uparrow 1.6750$ \\
\hline 9 & IPI00020599.1 & CALR, calreticulin precursor & $\uparrow 1.7062$ \\
\hline 10 & IPI00219219.3 & LGALS1, galectin-1 & $\uparrow 1.7379$ \\
\hline 11 & IPI00218918.5 & ANXA1, annexin A1 & $\uparrow 1.7379$ \\
\hline 12 & IPI00218733.6 & SOD1, superoxide dismutase & $\uparrow 1.7864$ \\
\hline 13 & IPI00010796.1 & $\mathrm{P} 4 \mathrm{HB}$, protein disulfide-isomerase precursor & $\uparrow 1.7864$ \\
\hline 14 & IPI00414283.5 & FN1, fibronectin 1 isoform 4 preproprotein & $\uparrow 1.8198$ \\
\hline 15 & IPI00298547.3 & PARK7, protein DJ-1 & $\uparrow 1.8198$ \\
\hline 16 & IPI00794402.1 & ARHGDIA, $28 \mathrm{kDa}$ protein & $\uparrow 1.8365$ \\
\hline 17 & IPI00219446.5 & PEBP1, phosphatidylethanolamine-binding protein 1 & $\uparrow 1.8879$ \\
\hline 18 & IPI00553177.1 & SERPINA1 & $\uparrow 1.9771$ \\
\hline 19 & IPI00029623.1 & PSMA6, proteasome subunit $\alpha$ type- 6 & $\uparrow 1.9952$ \\
\hline 20 & IPI00026314.1 & GSN, isoform 1 of gelsolin precursor & $\uparrow 2.0137$ \\
\hline 21 & IPI00479186.5 & PKM2 & $\uparrow 2.0700$ \\
\hline 22 & IPI00033494.3 & MRLC2, myosin regulatory light chain & $\uparrow 2.0700$ \\
\hline 23 & IPI00418471.6 & VIM, vimentin & $\uparrow 2.2492$ \\
\hline 24 & IPI00169383.3 & PGK1, phosphoglycerate kinase 1 & $\uparrow 2.2492$ \\
\hline 25 & IPI00396321.1 & LRRC59, leucine-rich repeat-containing protein 59 & $\uparrow 2.2696$ \\
\hline 26 & IPI00027947.6 & CTRL, chymotrypsin-like protease & $\uparrow 2.2696$ \\
\hline 27 & IPI00884105.1 & LAMP1 & $\uparrow 2.3337$ \\
\hline 28 & IPI00789605.1 & MYL6 & $\uparrow 2.3770$ \\
\hline 29 & IPI00219018.7 & GAPDH, glyceraldehyde-3-phosphate dehydrogenase & $\uparrow 2.3770$ \\
\hline 30 & IPI00021405.3 & LMNA, isoform A of lamin-A/C & $\uparrow 2.3770$ \\
\hline 31 & IPI00654755.3 & HBB, hemoglobin subunit $\beta$ & $\uparrow 2.3987$ \\
\hline 32 & IPI00024284.4 & HSPG2 & $\uparrow 2.4661$ \\
\hline 33 & IPI00020987.1 & PRELP, prolargin precursor & $\uparrow 2.4888$ \\
\hline 34 & IPI00871843.1 & TGM2, $81 \mathrm{kDa}$ protein & $\uparrow 2.5349$ \\
\hline 35 & IPI00418169.3 & ANXA2, annexin A2 isoform 1 & $\uparrow 2.5349$ \\
\hline 36 & IPI00291136.4 & COL6A1, collagen $\alpha-1(\mathrm{VI})$ chain & $\uparrow 2.5349$ \\
\hline 37 & IPI00009771.6 & LMNB2, lamin-B2 & $\uparrow 2.5349$ \\
\hline 38 & IPI00742225.1 & LOC646483, DNA-binding protein TAXREB107 isoform 1 & $\uparrow 2.5589$ \\
\hline 39 & IPI00297084.7 & DDOST & $\uparrow 2.6062$ \\
\hline 40 & IPI00216138.6 & TAGLN, transgelin & $\uparrow 2.6546$ \\
\hline 41 & IPI00025252.1 & PDIA3, protein disulfide-isomerase A3 & $\uparrow 2.6788$ \\
\hline 42 & IPI00414676.6 & HSP90AB1, heat-shock protein HSP 90- $\beta$ & $\uparrow 2.8843$ \\
\hline 43 & IPI00009904.1 & PDIA4, protein disulfide-isomerase A4 & $\uparrow 2.8843$ \\
\hline 44 & IPI00382696.1 & FLNB, isoform 2 of filamin-B & $\uparrow 2.9104$ \\
\hline 45 & IPI00022200.2 & COL6A3, $\alpha 3$ type VI collagen isoform 1 & $\uparrow 2.9922$ \\
\hline 46 & IPI00479145.2 & KRT19, type I cytoskeletal 19 & $\uparrow 3.0202$ \\
\hline 47 & IPI00792191.1 & GATM, glycine amidinotransferase & $\uparrow 3.0479$ \\
\hline 48 & IPI00872814.1 & Uncharacterized protein MSN (fragment) & $\uparrow 3.1328$ \\
\hline 49 & IPI00008274.7 & CAP1, adenylate cyclase-associated protein 1 & $\uparrow 3.1328$ \\
\hline 50 & IPI00887241.1 & LOC650788, 40S ribosomal protein S28 & $\uparrow 3.2206$ \\
\hline 51 & IPI00829626.1 & IGL@ protein & $\uparrow 3.2206$ \\
\hline 52 & IPI00220278.5 & MYL9, myosin regulatory light chain 2 & $\uparrow 3.2206$ \\
\hline
\end{tabular}


Table II. Continued.

\begin{tabular}{|c|c|c|c|}
\hline No. & Accession no. & Protein name & GMAH vs. NGM \\
\hline 53 & IPI00021766.5 & RTN4, isoform 1 of reticulon- 4 & $\uparrow 3.2510$ \\
\hline 54 & IPI00871932.1 & SPTBN1, $276 \mathrm{kDa}$ protein & $\uparrow 3.3422$ \\
\hline 55 & IPI00465431.7 & LGALS3, galectin-3 & $\uparrow 3.3422$ \\
\hline 56 & IPI00333541.6 & FLNA, filamin-A & $\uparrow 3.4674$ \\
\hline 57 & IPI00221226.7 & ANXA6, annexin A6 & $\uparrow 3.5323$ \\
\hline 58 & IPI00025465.1 & OGN, mimecan precursor & $\uparrow 3.5323$ \\
\hline 59 & IPI00013296.3 & RPS18 & $\uparrow 3.5651$ \\
\hline 60 & IPI00299145.9 & KRT6C, type II cytoskeletal 6C & $\uparrow 3.7665$ \\
\hline 61 & IPI00515087.2 & CTRB2, chymotrypsinogen B2 & $\uparrow 4.0933$ \\
\hline 62 & IPI00450768.7 & KRT17, type I cytoskeletal 17 & $\uparrow 4.0933$ \\
\hline 63 & IPI00745872.2 & ALB, isoform 1 of serum albumin precursor & $\uparrow 4.2070$ \\
\hline 64 & IPI00218914.5 & ALDH1A1, retinal dehydrogenase 1 & $\uparrow 4.8309$ \\
\hline 65 & IPI00027350.3 & PRDX2, peroxiredoxin-2 & $\uparrow 4.8309$ \\
\hline 66 & IPI00000874.1 & PRDX1, peroxiredoxin-1 & $\uparrow 4.8309$ \\
\hline 67 & IPI00887678.1 & LOC654188, peptidylprolyl isomerase A-like & $\uparrow 5.1520$ \\
\hline 68 & IPI00848226.1 & GNB2L1 & $\uparrow 5.3937$ \\
\hline 69 & IPI00883857.1 & HNRNPU & $\uparrow 5.4945$ \\
\hline 70 & IPI00744153.2 & Uncharacterized protein GCG & $\uparrow 5.8072$ \\
\hline 71 & IPI00020986.2 & LUM, lumican precursor & $\uparrow 5.8617$ \\
\hline 72 & IPI00010471.5 & LCP1, plastin-2 & $\uparrow 5.9172$ \\
\hline 73 & IPI00028030.3 & COMP, cartilage oligomeric matrix protein & $\uparrow 6.1958$ \\
\hline 74 & IPI00220271.3 & AKR1A1, alcohol dehydrogenase & $\uparrow 6.6050$ \\
\hline 75 & IPI00000690.1 & AIFM1, isoform 1 of apoptosis-inducing factor 1 & $\uparrow 6.6050$ \\
\hline 76 & IPI00296099.6 & THBS1, thrombospondin-1 precursor & $\uparrow 6.7935$ \\
\hline 77 & IPI00798430.1 & $\mathrm{TF}$, transferrin variant & $\uparrow 7.0472$ \\
\hline 78 & IPI00410241.2 & POSTN, periostin, osteoblast specific factor & $\uparrow 7.1124$ \\
\hline 79 & IPI00646304.4 & PPIB, peptidylprolyl isomerase B precursor & $\uparrow 7.3801$ \\
\hline 80 & IPI00022391.1 & APCS, serum amyloid P-component precursor & $\uparrow 7.3801$ \\
\hline 81 & IPI00021263.3 & YWHAZ, 14-3-3 protein $\zeta / \delta$ & $\uparrow 7.3801$ \\
\hline 82 & IPI00607708.3 & LDHA, isoform 2 of L-lactate dehydrogenase A chain & $\uparrow 8.3963$ \\
\hline 83 & IPI00749250.2 & ACTR2 $45 \mathrm{kDa}$ protein & $\uparrow 8.7108$ \\
\hline 84 & IPI00004457.3 & AOC 3 , membrane copper amine oxidase & $\uparrow 10.2775$ \\
\hline 85 & IPI00027463.1 & S100A6, protein S100 A6 & $\uparrow 10.3734$ \\
\hline 86 & IPI00215719.6 & RPL18, 60S ribosomal protein L18 & $\uparrow 10.7643$ \\
\hline 87 & IPI00014361.1 & TSTA3, GDP-L-fucose synthetase & $\uparrow 11.3766$ \\
\hline 88 & IPI00012750.3 & RPS25, 40S ribosomal protein $\mathrm{S} 25$ & $\uparrow 12.2399$ \\
\hline 89 & IPI00010414.4 & PDLIM1, PDZ and LIM domain protein 1 & $\uparrow 12.2399$ \\
\hline 90 & IPI00744375.1 & HLA-C & $\uparrow 12.7065$ \\
\hline 91 & IPI00399007.5 & IGHG2 & $\uparrow 13.5501$ \\
\hline 92 & IPI00291006.1 & MDH2 & $\uparrow 14.4509$ \\
\hline 93 & IPI00807428.1 & Putative uncharacterized protein & $\uparrow 16.8919$ \\
\hline 94 & IPI00738499.2 & FTL, ferritin light chain & $\uparrow 20.8768$ \\
\hline 95 & IPI00215965.2 & HNRNPA1 & $\uparrow 26.5252$ \\
\hline 96 & IPI00790262.1 & TTLL3 & $\uparrow 27.0270$ \\
\hline 97 & IPI00550991.3 & SERPINA3 & $\uparrow 32.4675$ \\
\hline 98 & IPI00015911.1 & DLD, dihydrolipoyl dehydrogenase & $\uparrow 38.7597$ \\
\hline 99 & IPI00060715.1 & KCTD12 & $\uparrow 39.0625$ \\
\hline 100 & IPI00465084.6 & DES, desmin & $\downarrow 0.0406$ \\
\hline 101 & IPI00396378.3 & HNRNPA2B1 & $\downarrow 0.0855$ \\
\hline 102 & IPI00514669.1 & SH3BGRL & $\downarrow 0.0991$ \\
\hline 103 & IPI00027827.2 & SOD3 & $\downarrow 0.1057$ \\
\hline 104 & IPI00025476.1 & AMY1B, pancreatic $\alpha$-amylase precursor & $\downarrow 0.1086$ \\
\hline
\end{tabular}


Table II. Continued.

\begin{tabular}{|c|c|c|c|}
\hline No. & Accession no. & Protein name & GMAH vs. NGM \\
\hline 105 & IPI00473011.3 & HBD, hemoglobin subunit $\delta$ & $\downarrow 0.1127$ \\
\hline 106 & IPI00847342.1 & KRT7, keratin 7 & $\downarrow 0.1148$ \\
\hline 107 & IPI00877792.1 & FGG, $50 \mathrm{kDa}$ protein & $\downarrow 0.1259$ \\
\hline 108 & IPI00815665.1 & PRSS1, PRSS1 protein & $\downarrow 0.1259$ \\
\hline 109 & IPI00011654.2 & TUBB, tubulin $\beta$ chain & $\downarrow 0.1306$ \\
\hline 110 & IPI00021885.1 & FGA, isoform 1 of fibrinogen $\alpha$ chain precursor & $\downarrow 0.1318$ \\
\hline 111 & IPI00009634.1 & SQRDL & $\downarrow 0.1803$ \\
\hline 112 & IPI00478003.1 & $\mathrm{A} 2 \mathrm{M}, \alpha_{2}$-macroglobulin precursor & $\downarrow 0.2014$ \\
\hline 113 & IPI00867509.1 & CORO1C, coronin-1C_i3 protein & $\downarrow 0.2291$ \\
\hline 114 & IPI00642455.2 & THBS2, thrombospondin 2 & $\downarrow 0.2291$ \\
\hline 115 & IPI00000105.4 & MVP, major vault protein & $\downarrow 0.2377$ \\
\hline 116 & IPI00027720.1 & PNLIP, pancreatic triacylglycerol lipase precursor & $\downarrow 0.2421$ \\
\hline 117 & IPI00140420.4 & SND1 & $\downarrow 0.2805$ \\
\hline 118 & IPI00515061.3 & HIST1H2BJ, histone H2B type 1-J & $\downarrow 0.2884$ \\
\hline 119 & IPI00410714.5 & HBA1, hemoglobin subunit $\alpha$ & $\downarrow 0.2911$ \\
\hline 120 & IPI00295663.1 & ELA3A, elastase-3A precursor & $\downarrow 0.2965$ \\
\hline 121 & IPI00298497.3 & FGB, fibrinogen $\beta$ chain precursor & $\downarrow 0.2992$ \\
\hline 122 & IPI00759832.1 & YWHAB, isoform short of $14-3-3$ protein $\beta / \alpha$ & $\downarrow 0.3020$ \\
\hline 123 & IPI00003527.5 & SLC9A3R1 & $\downarrow 0.3221$ \\
\hline 124 & IPI00788782.1 & ATP1A $3, \mathrm{Na}^{+} / \mathrm{K}^{+}$-ATPase $\alpha 3$ subunit variant & $\downarrow 0.3404$ \\
\hline 125 & IPI00028908.3 & NID2, nidogen-2 precursor & $\downarrow 0.3532$ \\
\hline 126 & IPI00186290.6 & EEF2, elongation factor 2 & $\downarrow 0.3698$ \\
\hline 127 & IPI00010779.4 & TPM4, isoform 1 of tropomyosin $\alpha-4$ chain & $\downarrow 0.3767$ \\
\hline 128 & IPI00873444.1 & UBC, RPS27A $79 \mathrm{kDa}$ protein & $\downarrow 0.3837$ \\
\hline 129 & IPI00156689.3 & VAT1 & $\downarrow 0.3945$ \\
\hline 130 & IPI00178926.2 & IGJ, immunoglobulin J chain & $\downarrow 0.3981$ \\
\hline 131 & IPI00021827.3 & DEFA3, neutrophil defensin 3 precursor & $\downarrow 0.3981$ \\
\hline 132 & IPI00337741.4 & APEH, acylamino-acid-releasing enzyme & $\downarrow 0.4055$ \\
\hline 133 & IPI00292530.1 & ITIH1, inter- $\alpha$-trypsin inhibitor heavy chain H1 & $\downarrow 0.4169$ \\
\hline 134 & IPI00031522.2 & HADHA, trifunctional enzyme subunit $\alpha$ & $\downarrow 0.4207$ \\
\hline 135 & IPI00426051.3 & Putative uncharacterized protein DKFZp686C15213 & $\downarrow 0.4246$ \\
\hline 136 & IPI00009027.1 & REG1A, lithostathine-1- $\alpha$ precursor & $\downarrow 0.4246$ \\
\hline 137 & IPI00300725.7 & KRT6A, type II cytoskeletal 6A & $\downarrow 0.4365$ \\
\hline 138 & IPI00555744.6 & RPL14 protein & $\downarrow 0.4529$ \\
\hline 139 & IPI00465361.4 & RPL13, 60S ribosomal protein L13 & $\downarrow 0.4571$ \\
\hline 140 & IPI00843810.2 & CEL, carboxyl ester lipase & $\downarrow 0.4699$ \\
\hline 141 & IPI00024933.3 & RPL12, 60S ribosomal protein L12 & $\downarrow 0.4966$ \\
\hline 142 & IPI00845263.1 & FN1, fibronectin 1 isoform 2 preproprotein & $\downarrow 0.5012$ \\
\hline 143 & IPI00449920.1 & IGHA1, highly similar to $\operatorname{Ig} \alpha-1$ chain $\mathrm{C}$ region & $\downarrow 0.5105$ \\
\hline 144 & IPI00289862.3 & SCRN1, secernin-1 & $\downarrow 0.5105$ \\
\hline 145 & IPI00002745.1 & CTSZ, cathepsin Z precursor & $\downarrow 0.5152$ \\
\hline 146 & IPI00005924.4 & PNLIPRP2, pancreatic lipase-related protein 2 & $\downarrow 0.5297$ \\
\hline 147 & IPI00873137.1 & COL1A2, $130 \mathrm{kDa}$ protein & $\downarrow 0.5346$ \\
\hline 148 & IPI00783512.1 & Reversed PTPRN2 110 kDa protein & $\downarrow 0.5346$ \\
\hline 149 & IPI00552768.1 & TXN, thioredoxin & $\downarrow 0.5346$ \\
\hline 150 & IPI00294380.5 & PCK2 & $\downarrow 0.5395$ \\
\hline 151 & IPI00007765.5 & HSPA9, stress-70 protein, mitochondrial precursor & $\downarrow 0.5445$ \\
\hline 152 & IPI00472724.1 & EEF1AL3, elongation factor 1 - $\alpha$-like 3 & $\downarrow 0.5495$ \\
\hline 153 & IPI00297646.4 & COL1A1, collagen $\alpha-1(\mathrm{I})$ chain & $\downarrow 0.5495$ \\
\hline 154 & IPI00307162.2 & VCL, isoform 2 of vinculin & $\downarrow 0.5649$ \\
\hline 155 & IPI00009826.2 & CPB1, carboxypeptidase B precursor & $\downarrow 0.5649$ \\
\hline 156 & IPI00003362.2 & HSPA5 protein & $\downarrow 0.5649$ \\
\hline
\end{tabular}


Table II. Continued.

\begin{tabular}{llll}
\hline No. & Accession no. & \multicolumn{1}{c}{ Protein name } & GMAH vs. NGM \\
\hline 157 & IPI00305461.2 & ITIH2, inter- $\alpha$-trypsin inhibitor heavy chain H2 & $\downarrow 0.5754$ \\
158 & IPI00027497.5 & GPI, glucose-6-phosphate isomerase & $\downarrow 0.5971$ \\
159 & IPI00061005.4 & ERP27, endoplasmic reticulum-resident protein ERp27 & $\downarrow .6138$ \\
160 & IPI00298994.6 & TLN1, talin-1 & $\downarrow .6194$ \\
161 & IPI00026302.3 & RPL31, 60S ribosomal protein L31 & $\downarrow .6194$ \\
162 & IPI00856098.1 & p180/ribosome receptor & $\downarrow 0.6252$ \\
163 & IPI00216134.3 & TPM1, tropomyosin 1 $\alpha$ chain isoform 7 & $\downarrow .6252$ \\
164 & IPI00009823.3 & CPA1, carboxypeptidase A1 precursor & $\downarrow$ \\
165 & IPI00009867.3 & KRT5, type II cytoskeletal 5 & $\downarrow 0.6486$ \\
\hline
\end{tabular}

S100A6 and SOD3 are highlighted in bold. NGM, normal gastric mucosa; GMAH, gastric mucosal atypical hyperplasia; $\uparrow$, upregulation of expression in GMAH mesenchyma; $\downarrow$, downregulation of expression in GMAH mesenchyma.

Table III. Expression levels of S100A6 and SOD3 proteins in the NGM and GMAH tissues.

\begin{tabular}{|c|c|c|c|c|c|}
\hline \multirow[b]{2}{*}{ Protein } & \multirow[b]{2}{*}{$\mathrm{n}$} & \multicolumn{3}{|c|}{ Score } & \multirow[b]{2}{*}{ Positive rate, $\%$} \\
\hline & & Low (-) & Moderate (+) & High $(+++)$ & \\
\hline \multicolumn{6}{|l|}{ SOD3 } \\
\hline NGM & 20 & 8 & 8 & 4 & 60.00 \\
\hline GMAH & 20 & 14 & 4 & 2 & $30.00^{\mathrm{a}}$ \\
\hline \multicolumn{6}{|l|}{ S100A6 } \\
\hline NGM & 20 & 13 & 4 & 3 & 35.00 \\
\hline GMAH & 20 & 7 & 11 & 2 & $65.00^{\mathrm{a}}$ \\
\hline
\end{tabular}

aP $<0.05$, GMAH vs. NGM tissues. SOD3, superoxide dismutase 3; S100A6, S100 calcium-binding protein A6; NGM, normal gastric mucosa; GMAH, gastric mucosal atypical hyperplasia.

expression of S100A6 and SOD3 was analyzed by western blotting and immunohistochemical staining techniques, and was identified to be significantly different and associated with tumorigenesis. These results were consistent with the results of quantitative proteomics in the present study.

S100A6 is a member of the S100 protein family (15). S100A6 has a number of biological functions, including participating in the degradation and ubiquitination of $\beta$-catenin, promoting apoptosis, interacting with extracellular matrix proteins, enhancing cell metabolism and skeleton depolymerization, participating in endocytosis and exocytosis, adjusting enzyme activity, inhibiting protein kinase $\mathrm{C}$-mediated phosphorylation and participating in gene transcription $(16,17)$. A number of studies have demonstrated that S100A6 is also associated with the occurrence and development of tumors and is upregulated in several tumors, including ovarian cancer, colorectal cancer, pancreatic cancer, liver cancer, malignant melanoma and osteosarcoma $(18,19)$. According to the results of the present study, S100A6 is upregulated in the GMAH stroma. This protein may contribute to the malignant transformation of epithelial cells of gastric mucosa and promote cell invasion and metastasis. The S100A6 protein may be a potential biomarker for monitoring malignant cell transformation.

Mammalian SODs have three subtypes, namely the cytoplasmic SOD (CuZnSOD or SOD1), mitochondrial SOD (MnSOD or SOD2) and extracellular SOD (EC-SOD or SOD3) (20). SOD3 serves an important function in maintaining the oxidation balance that prevents nuclear DNA and protein oxidative damage in the extracellular matrix and nucleus (21). Previous studies have identified that the level of SOD3 was decreased in a variety of tumors, including lung, breast and thyroid cancer, and renal cell carcinoma $(10,22)$. SOD3 is widely expressed in normal tissues; low or no expression of SOD3 causes an imbalance in the extracellular redox environment and cancer occurs more frequently in an imbalanced environment (23). Therefore, a low or no expression of SOD3 may be a risk factor for malignant cell transformation (24). The results of the present study demonstrated that SOD3 was downregulated in GMAH stroma, which resulted in DNA damage in gastric mucosa epithelial cells and GC. Therefore, the early detection of SOD3 may predict the occurrence of GC.

As the tumor microenvironment serves a critical function in GC occurrence and development, it important to identify 
A S100A6 (LQDAEIAR $M / Z Z=2)$
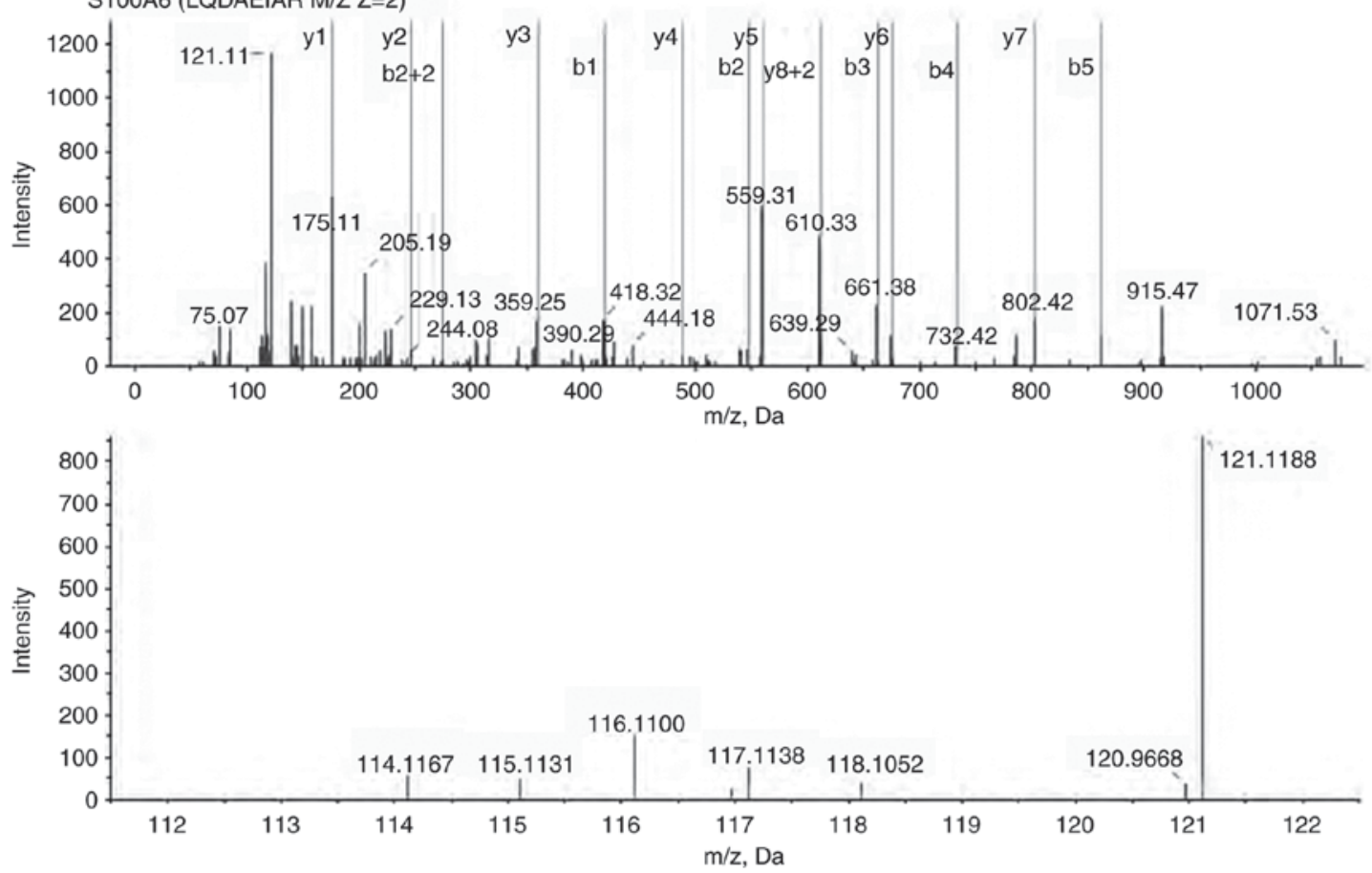

B SOD3 (VTGVVLFR M/Z Z $=3$ )
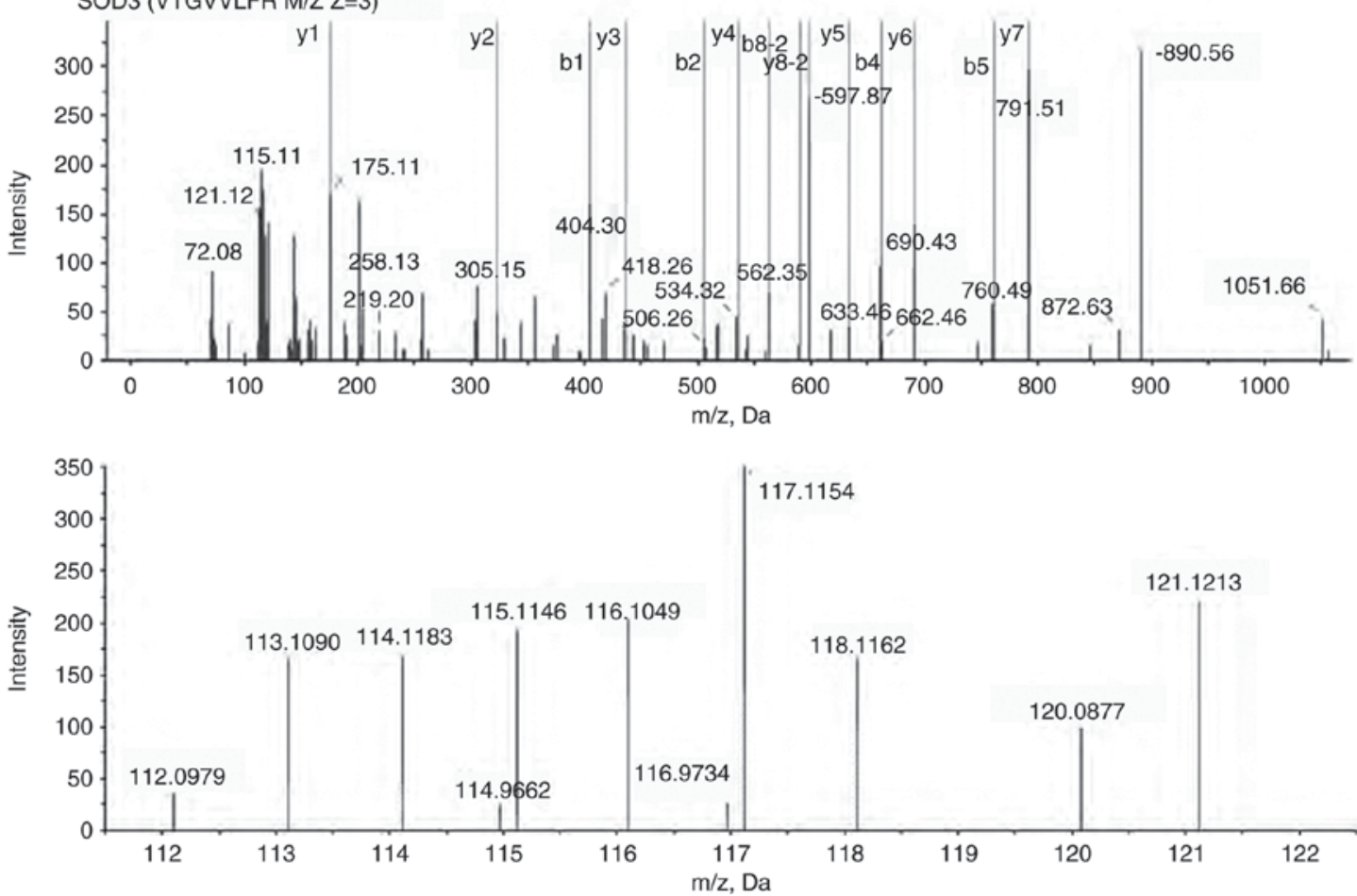

Figure 2. Mass spectrometry and quantification of S100A6 and SOD3 proteins. (A) Top: The peptide mass fingerprint and sequence LQDAEIAR allowed the identification of S100A6. Bottom: The released iTRAQ reporter ions provided the relative quantification of S100A6 from the mesenchyma of NGM and GMAH tissues evaluated. (B) Top: The peptide mass fingerprint and sequence VTGVVLFR allowed the identification of SOD3. Bottom: The released iTRAQ reporter ions provide the relative quantification of SOD3 from the two tissues evaluated. S100A6, S100 calcium-binding protein A6; SOD3, superoxide dismutase 3; iTRAQ, isobaric tags for relative and absolute quantitation.

the proteins present in the GMAH microenvironment. The present study identified a total of 165 differentially expressed proteins in GMAH stroma. These data will further clarify the molecular mechanisms of GC occurrence as well as potentially serving as prognostic markers for the early detection and diagnosis of GC.

\section{Acknowledgements}

The authors would like to thank Dr Qiang Zhao from The First Affiliated Hospital of University of South China (Hengyang, China) for his help in collecting specimens. The authors would also like to thank Professor Zhao-Yang Luo 
A

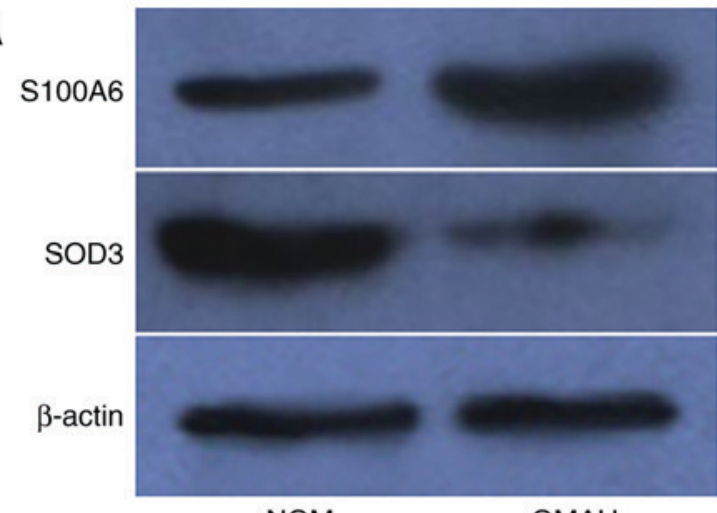

NGM

GMAH
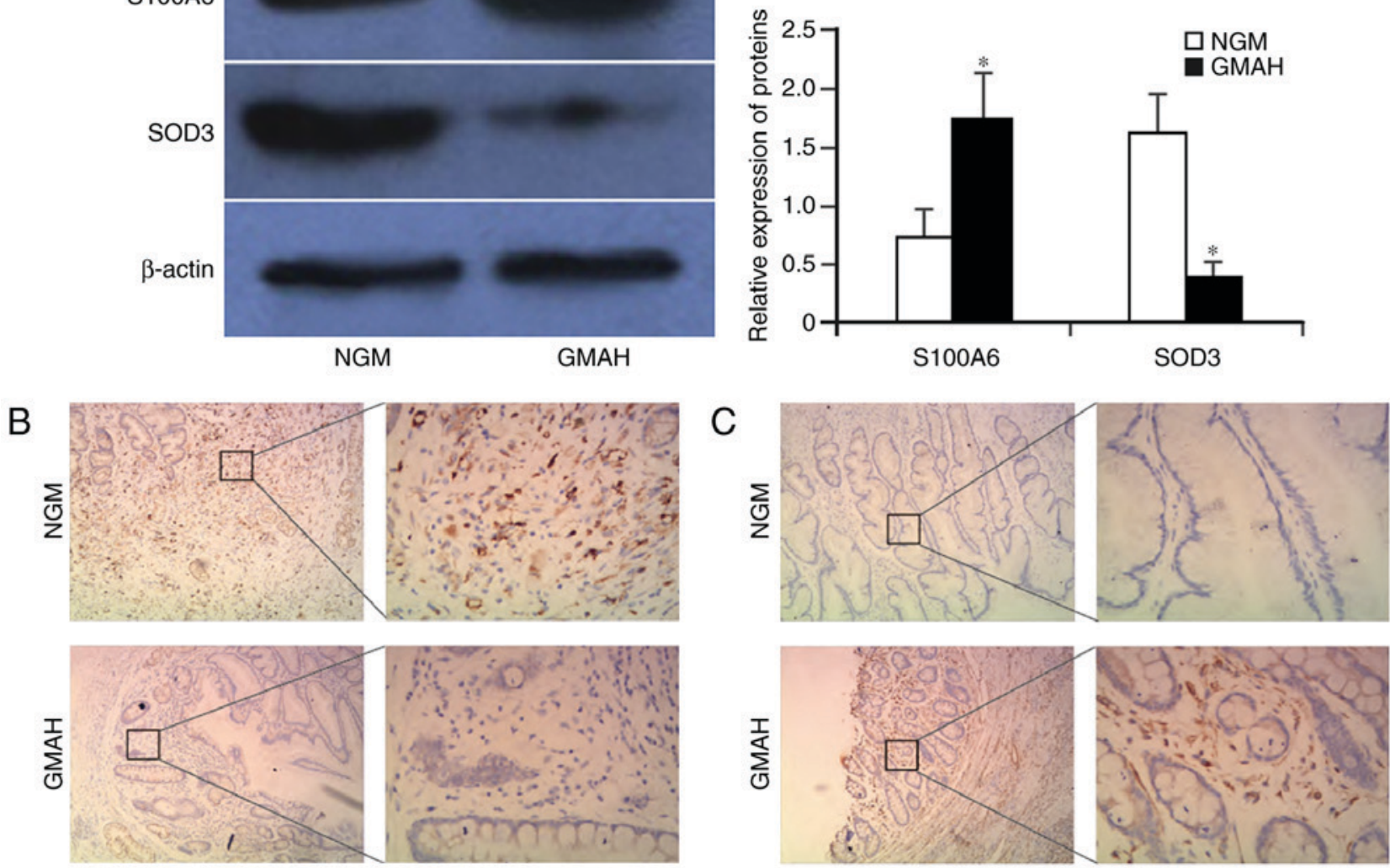

Figure 3. Expression of S100A6 and SOD3 proteins in the mesenchyma of NGM and GMAH tissues. (A) The expression of S100A6 and SOD3 proteins in NGM and GMAH mesenchymal tissues were detected by western blot analysis. Expression levels of proteins in two tissues were determined by densitometric analysis ( $\mathrm{n}=3$; " $\mathrm{P}<0.01$ vs. NGM). (B) Immunohistochemistry revealed the expression of the S100A6 protein in NGM and GMAH mesenchyma. Original magnification, $\mathrm{x} 10$ (left) and $\mathrm{x} 40$ (right). (C) Immunohistochemistry revealed the expression of the SOD3 protein in NGM and GMAH mesenchyma. Original magnification, x10 (left) and x40 (right). S100A6, S100 calcium-binding protein A6; SOD3, superoxide dismutase 3; NGM, normal gastric mucosa; GMAH, gastric mucosal atypical hyperplasia.
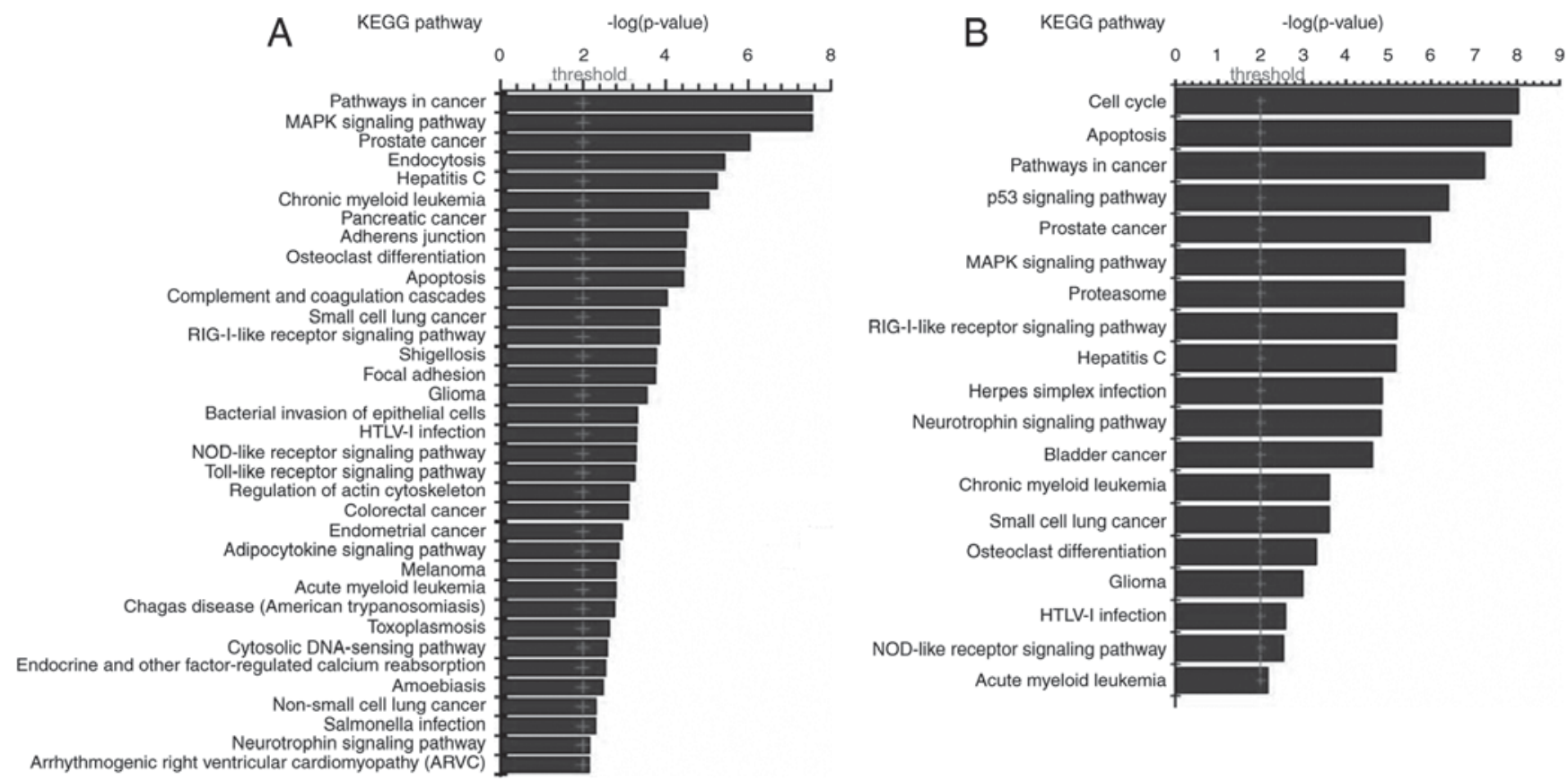

Figure 4. KEGG signal pathway analysis of differentially expressed proteins analyzed using Cytoscape software. (A) The signal pathway involved in the proteins that are upregulated in GMAH compared with NGM tissues. (B) The signal pathway involved in the proteins that are downregulated in GMAH compared with NGM tissues. KEGG, Kyoto Encyclopedia of Genes and Genomes; NGM, normal gastric mucosa; GMAH, gastric mucosal atypical hyperplasia; MAPK, mitogen-activated protein kinase; RIG, retinoic acid-induced gene; HTLV, human T-lymphotropic virus; NOD, nucleotide-binding oligomerization domain. 
and Professor Xiu-Tian Zhou from the Cancer Research Institute of the University of South China (Hengyang, China) for their assistance in the diagnosis of specimens.

\section{Funding}

The present study was supported by the Hunan Provincial Innovation Foundation For Postgraduates (grant no. CX2016B478), the Doctoral Research Start-Up Fund of the University of South China (grant no. 2016XQD21), the Hunan Provincial Groundbreaking Platform Open Fund of the University of China (grant no. 10K052, 12K094 and 13K083), the Hunan Provincial Education Department Foundation of China (grant nos. 11C1112 and 12C0340), the Hunan Provincial Health Department Foundation of China (grant nos. B2013-048 and 2014-163) and the Construct Program of the Key Discipline in Hunan Province of China (2011-76).

\section{Availability of data and materials}

All data generated or analyzed during this study are included in this published article.

\section{Authors' contributions}

ZWZ and CYL conceived and designed the experiments. HLZ, WM and CJL performed the experiments. LH and CSL analyzed the data. HLZ and ZWZ wrote the paper. All authors read and approved the manuscript.

\section{Ethics approval and consent to participate}

All patients involved in this study provided written informed consent, and the present study was approved by the Medical Ethics Committee of University of South China. Written informed consent was obtained from all participants.

\section{Consent for publication}

All patients provided their written informed consent for the publication of their data.

\section{Competing interests}

The authors declare that they have no conflicts of interest.

\section{References}

1. Chen W, Zheng R, Baade PD, Zhang S, Zeng H, Bray F, Jemal A, $\mathrm{Yu}$ XQ and He J: Cancer statistics in China, 2015. CA Cancer J Clin 66: 115-132, 2016.

2. Lim B, Kim JH, Kim M and Kim SY: Genomic and epigenomic heterogeneity in molecular subtypes of gastric cancer. World J Gastroenterol 22: 1190-1201, 2016.

3. Correa P: Human gastric carcinogenesis: A multistep and multifactorial process-First American Cancer Society Award lecture on cancer epidemiology and prevention. Cancer Res 52: 6735-6740, 1992.

4. Kohlhapp FJ, Mitra AK, Lengyel E and Peter ME: MicroRNAs as mediators and communicators between cancer cells and the tumor microenvironment. Oncogene 34: 5857-5868, 2015.
5. Rodvold JJ and Zanetti M: Tumor microenvironment on the move and the Aselli connection. Sci Signal 9: fs13, 2016.

6. Langley RR and Fidler IJ: The seed and soil hypothesis revisited-the role of tumor-stroma interactions in metastasis to different organs. Int J Cancer 128: 2527-2535, 2011.

7. Chin AR and Wang SE: Cancer tills the premetastatic field: Mechanistic basis and clinical implications. Clin Cancer Res 22: 3725-3733, 2016.

8. Amin MB, Edge SB, Greene FL, Byrd DR, Brookland RK, Washington MK, Gershenwald JE, Compton CC, Hess KR, Sullivan DC, et al: AJCC cancer staging manual. 8th ed. New York: Springer 203-220, 2016.

9. Wang XH, Du H, Li L, Shao DF, Zhong XY, Hu Y, Liu YQ, Xing XF, Cheng XJ, Guo T, et al: Increased expression of S100A6 promotes cell proliferation in gastric cancer cells. Oncol Lett 13: 222-230, 2017.

10. Kim J, Mizokami A, Shin M, Izumi K, Konaka H, Kadono Y, Kitagawa Y, Keller ET, Zhang J and Namiki M: SOD3 acts as a tumor suppressor in PC-3 prostate cancer cells via hydrogen peroxide accumulation. Anticancer Res 34: 2821-2831, 2014.

11. Yan M and Jurasz P: The role of platelets in the tumor microenvironment: From solid tumors to leukemia. Biochim Biophys Acta 1863: 392-400, 2016.

12. Justus CR, Sanderlin EJ and Yang LV: Molecular connections between cancer cell metabolism and the tumor microenvironment. Int J Mol Sci 16: 11055-11086, 2015.

13. Linton SS, Sherwood SG, Drews KC and Kester M: Targeting cancer cells in the tumor microenvironment: Opportunities and challenges in combinatorial nanomedicine. Wiley Interdiscip Rev Nanomed Nanobiotechnol 8: 208-222, 2016.

14. Wu AA, Drake V,Huang HS, Chiu S and Zheng L: Reprogramming the tumor microenvironment: Tumor-induced immunosuppressive factors paralyze T cells. Oncoimmunology 4: e1016700, 2015.

15. Schneider G and Filipek A: S100A6 binding protein and Siah-1 interacting protein (CacyBP/SIP): Spotlight on properties and cellular function. Amino Acids 41: 773-780, 2011.

16. Leśniak W, Słomnicki ŁP and Filipek A: S100A6-new facts and features. Biochem Biophys Res Commun 390: 1087-1092, 2009.

17. Kasacka I, Piotrowska Ż, Filipek A and Majewski M: Influence of doxazosin on biosynthesis of S100A6 and atrial natriuretic factor peptides in the heart of spontaneously hypertensive rats. Exp Biol Med (Maywood) 241: 375-381, 2016.

18. Zhang J, Zhang K, Jiang X and Zhang J: S100A6 as a potential serum prognostic biomarker and therapeutic target in gastric cancer. Dig Dis Sci 59: 2136-2144, 2014.

19. Duan L, Wu R, Zou Z, Wang H, Ye L, Li H, Yuan S, Li X, Zha H, Sun $\mathrm{H}$, et al: S100A6 stimulates proliferation and migration of colorectal carcinoma cells through activation of the MAPK pathways. Int J Oncol 44: 781-790, 2014.

20. Che M, Wang R, Li X, Wang HY and Zheng XFS: Expanding roles of superoxide dismutases in cell regulation and cancer. Drug Discov Today 21: 143-149, 2016.

21. Wang YD, Chen WD, Li C, Guo C, Li Y, Qi H, Shen H, Kong J, Long X, Yuan F, et al: Farnesoid X receptor antagonizes JNK signaling pathway in liver carcinogenesis by activating SOD3. Mol Endocrinol 29: 322-331, 2015.

22. Xu D, Li Y, Li X, Wei LL, Pan Z, Jiang TT, Chen ZL, Wang C, Cao WM, Zhang X, et al: Serum protein S100A9, SOD3, and MMP9 as new diagnostic biomarkers for pulmonary tuberculosis by iTRAQ-coupled two-dimensional LC-MS/MS. Proteomics 15: 58-67, 2015

23. Griess B, Tom E, Domann F and Teoh-Fitzgerald M: Extracellular superoxide dismutase and its role in cancer. Free Radic Biol Med 112: 464-479, 2017.

24. O'Leary BR, Fath MA, Bellizzi AM, Hrabe JE, Button AM, Allen BG, Case AJ, Altekruse S, Wagner BA, Buettner GR, et al: Loss of SOD3 (EcSOD) expression promotes an aggressive phenotype in human pancreatic ductal adenocarcinoma. Clin Cancer Res 21: 1741-1751, 2015.

This work is licensed under a Creative Commons Attribution-NonCommercial-NoDerivatives 4.0 International (CC BY-NC-ND 4.0) License. 